\title{
Universities Computer Curriculum Reform under the Perspective of Computational Thinking
}

\author{
Yuan Chen ${ }^{1, a}$ \\ ${ }^{1}$ Nanyang Medical College, Nanyang, Henan, 473000 \\ a email
}

Keywords: Computational Thinking; Basic Computer Course; Algorithm; Database; Network

\begin{abstract}
Computational thinking is one of the hot current computer education concern. This paper describes the connotation of computational thinking and their current progress, and from the perspective of computational thinking to sort out basic computer course content university, and algorithms, databases and network technology preliminary analysis, to solve practical problems for the other areas of expertise to provide inspiration and the method of Reference.
\end{abstract}

\section{Introduction}

The current basic computer course content Colleges generally made basic knowledge of computer hardware and software, databases, multimedia, network and information security, and several other modules. Since part of the teaching content and high school information technology curriculum content duplication, resulting in little interest in learning, lessons and other academic departments to reduce the adverse situation. So, where is the crux of the problem then. Computer basic course and advanced mathematics, English as a fundamental part of quality university education or general education, universal concepts that can be taught students computational thinking, methods and techniques, such as algorithm thinking, thinking and computing systems protocol thinking and the application of a wide range of database and network technology. Trying to guide students consciously computational thinking throughout the study and work them, and understanding of computing in extending human imagination and creativity and other aspects of a huge role.

Computational thinking as one of the basic form of human scientific thinking, should belong to a specialized field of scientific thinking. Currently the concept of computational thinking has been widely recognized by the Carnegie Mellon University, USA • proposed by Professor Jeannette Wing, that computational thinking is the use of the fundamental concepts of computer science to solving problems, designing systems and understanding human behavior, including covering computer a series of scientific thinking activities breadth. Computational thinking is the essence of abstraction and automation, it reflects the fundamental problem of the calculation, namely what can be effectively automated. Education refers to the Commission attaches great importance to computational thinking in recent years, held a special meeting to study and investigate the computational thinking at the University of Education Position computational thinking and creative ability of students an important role in published "C9 League (C9 ) computer basic Education development strategy of the joint statement, "the basis for determining the direction of reform in Teaching computational thinking as the core of a computer, recently also received the support of the Ministry of Education project.

In order to calculate the thinking ability of students, the need for basic computer course content to re-examine and comb. From the perspective of computational thinking of starting, basic computer teaching content should include: computational thinking and computational theory, algorithm basis and common language, computer hardware and software technology, computing and other social and vocational topics. According to calculations, the concept of thinking proposed by Professor Jeannette Wing, which should include problem solving, system design and understanding of human behavior in three aspects. From the following three aspects, it is in basic computer course in how to use computational thinking to solve real problems in different areas of expertise. 


\section{Algorithm for Solving Problems}

Algorithm is computer science methodology nature of most core concept, also known as the soul of the computer. Although the algorithm is to calculate theoretical knowledge category, but the computer to solve any problems must rely on algorithms. Thus, basic computer courses at the University of algorithms can be added to improve the concept of structured thinking ability of students to deal with and solve problems. So how do you train the algorithm thinking it? The answer is to grasp the logic behind the algorithm. Different algorithms strategy seemingly different, but in reality the same strain, or from a higher-level perspective is the same way of thinking. Each algorithm design strategy as a method of problem solving can be applied to many areas of expertise, computational thinking has obvious characteristics.

Divide and conquer strategy. The basic idea of divide and conquer strategy to solve the problem is a big problem difficult to solve directly, broken down into a number of smaller sub-problems, in order to divide and conquer, divide and rule. If the child is still relatively big problem, divide and conquer algorithm can be used repeatedly, until the last sub-problems can be directly obtained their results. Divide and conquer strategy is to work to solve common problems a way to learn thinking and life, it has been widely used in organizational management and military fields. For example, a large corporate sales, because of its many products of high quality and very popular, the headquarters will be established around the branches (subsidiaries), which it implies Divide and Conquer.

Dynamic programming. The basic idea of dynamic programming problems are to be solved the problem into several interrelated sub-problems, then press the bottom-up sequence deduced solution to the original problem. By storing sub-problem

Solutions can be avoided in the solution process is repeated several times to solve the same problem child, which can improve computational efficiency of the algorithm. Dynamic programming algorithm is essentially combining ideological divide and conquer and redundancy solutions. Dynamic programming has been widely used in economic management, production scheduling, engineering and other aspects of optimal control, the shortest route, inventory management, resource allocation, equipment updating, sorting and loading problems using dynamic programming algorithm more convenient.

The greedy algorithm. Greedy algorithm to solve the problem of the basic idea is to solve the problem to be broken down into several sub-problems solved step by step, and each step is always to make the best choice for the current, in order to get the optimal solution problem. Greedy algorithm to obtain the optimal solution for each of its local sub-problems, and then integrated into the various local optima solution of the problem. It embodies a "Gordian knot" thinking to maximize current and local interests-oriented problem solving strategy is simple, has broad applicability.

\section{Database Design}

Database technology, computer science and technology is one of the most widely used technology, it has created a CW Bachman, EF Codd and James Gray three Turing Award winner, and led to a huge software industry - database management system products and solution. Database research has a remarkable productivity, generate an annual information industry tens of billions of dollars in revenue, economic development has great role in promoting. Database design for a given application environment, the design optimization of database logic model and physical structure, and thus establish a database and applications, to enable it to efficiently store and manage data, to meet a variety of user applications, including data and information management requirements operating requirements. Information management requirements should be stored in the database and management of data objects, data manipulation requirements are additions and deletions to the data object, query and statistical operations. Achievement of database systems to promote the progress of research in many fields, but also on industry and government management has brought enormous support. For example, listed companies database system is developed by the China Securities 
newspaper, which mainly provide comprehensive, accurate and authoritative information to the stock market listed companies substantially, while providing data services to Securities Daily and securities regulators. Through a Web browser or a search, you can analyze the data of listed companies, which found that most investment value of the company. Again, all levels of government can be established covering the social and economic space within the jurisdiction of the database, which contains the basic spatial data, natural resources and economic and social data resources, to provide timely and comprehensive information for government macroeconomic management, improve the administration of the public sector effectiveness.

\section{Understanding of Network Behavior}

Internet is considered one of the greatest inventions in human history, it revolutionized human communication and thinking patterns. But the network also gave rise to a number of security risks, there are some malicious programs that automatically log into the system by sending a lot of spam and try to deal. To solve this problem have been proposed automatic distinction between computer and human Turing test (Completely Automated Public Turing Test to Tell Computers and Humans Apart, called CAPTCHA), it can continue to be a judge, "it is a human or a machine" test program . According to statistics, CAPTCHA test customers worldwide, every day there are about 200 million, if each takes about 10 seconds. So every day more than 500,000 hours are for such calculations. That is, the global Internet each day to pay such a high price to prove that they are human beings, not machines. So, you can take advantage of users spend on the CAPTCHA test effort that will benefit the information society development goals? This is the original intention reCAPTCHA project - dedicated to digital print media. In order to facilitate faster and broader dissemination of knowledge, a lot of books and newspapers need to be digitized. reCAPTCHA is the use of CAPTCHA principle, by means of the human brain is difficult to identify the characters to identify technologies. That is, reCAPTCHA can not only anti-spam, and can also help with digitization. reCAPTCHA works: a test requires the user to identify the two words, one wants to digitize books from the interception out another system to know the correct answer. One of two answers given by the user matches the known correct answers, even pass the test. The word to be digitized will be randomly sent more tests in a large number of test results are aggregated, you can get the exact number of words digitized results.

Many well-known sites have to start from the original CAPTCHA reCAPTCHA. In less than 11 months time, the New York Times completed from 1851 to 1980, all digitized content. This is the power of the network, the Internet is the crystallization of wisdom and collective human behavior, but also a way of understanding human behavior computational thinking. Since the calculation of basic scientific thinking is a way of thinking, so the basic computer training course should imperceptibly and students computational thinking ability in the usual teaching, further training and improve their sense of innovation and creativity. Ability computational thinking throughout the process of university teaching computer systems engineering, computational thinking exists in many branches of computer, so the computer will be able to fully meet the basic course students computing strategy thinking ability is unrealistic. This requires that when setting the course content, not only to fully consider the relationship between the various branches of the computer, but also need to focus on computational thinking and its application in various areas of expertise, so that between course content, curriculum and curriculum have coherence between .

Control college English teaching for three years continuous line, that first and second grade is the foundation of English, is a third-year English or bilingual teaching, if college computer education is not just rely on 1 to 2 basic computer courses, but with the follow-up to various professional courses close coordination, so that a four-year college computer education continuous line, the students' computer skills will have a qualitative leap in computational thinking ability will fall into place. Thus, basic computer courses and how to follow-up with various professional computer-related courses seamless is an urgent problem to be solved, it is worth further exploration. 


\section{References}

[1] Zhu Yazong computational thinking [J] Computer Science, 2009,36 (4): 53-55,93.

[2] Jeannette M. Wing Computational Thinking [J] Communications of the ACM, 2006, 49 (3): 33-35.

[3] Dong Rongsheng. computer computational thinking and methodology [J] Computer Science, 2009,36 (4): 1-4,42.

[4]. Basic computer teaching development strategy of the Joint Declaration C9 League (C9) [J] Chinese university teaching, 2010 (9): 4

[5] Chen Guoliang. computational thinking and Computer Basic Education in University [J] Chinese university education, 2011 (1): 7-11.

[6] Zhou Hengming. Algorithm Road [M] Beijing: Mechanical Industry Press, 2011.

[7] Zhao Lingzhong. algorithm design strategies and computational thinking [J] Technology and Enterprise Development, 2010 (8): 43-45.

[8] Wang Shan. databases such as research and development of new technologies Web era [A] // China Computer Science and Technology Development Report (2006) [C] Beijing: Tsinghua University Press, 2007. 